\title{
Research and Implementation of Innovated Rehabilitation Robotics Manipulators
}

\author{
Chin-Wen Chuang \\ Dept. of Electrical Engineering \\ I-Shou University, \\ Kaohsiung, Taiwan
}

\author{
Meng-Hsiu Wu \\ Dept. of Electrical Engineering \\ I-Shou University, \\ Kaohsiung, Taiwan
}

\author{
Kuan-Yu Chen \\ Dept. of Electrical Engineering \\ I-Shou University, \\ Kaohsiung, Taiwan
}

\begin{abstract}
The Stroke has become the top ten leading cause of death. The serious patient will pass away. The light patient of stroke will lose the ability of one-side limb action. It will cause a lot of inconvenience for the caregivers and the family members. At the same time, it will be the burden on society. Based on the above reason, an intelligent wearable rehabilitation robotics is developed in this manuscript. In the golden recovery period, using the mechanical power to help the patient to repeat the flexion and stretching rehabilitation. It can effectively avoid the limb contracture. At the same time, in order to avoid the sports injuries, the EMG (electromyography) is used as feedback signal. It will detect the situation of muscle of the patient. Then, according to the situation, the robotics will automatically tune the travel length of the rehabilitation. Finally, prototype of the rehabilitation robotics will be implemented. The operation of the robotics will be shown in this manuscript.
\end{abstract}

\section{KEYWORD: Rehabilitation, EMG, Robotics}

\section{INTRODUCTION}

Due to the increasing of the social pressure from the industrial environment, the birthing rate decreases year by year. Taiwan slowly toward the aging society. The disease will cause large load for the elder society. Hence, the elder's healthy care is the important research. In theses elder diseases, the cerebral vascular accident is the major disease. If the one stroke, much sequel will happen, such as Hemiparalysis of limbs, sensory loss or abnormal, ataxia, unilateral limb weakness, involuntary movements, spasticity, etc.
Usually, the stroke is divided into three phases; those are acute phase, recovery phase and stationary phase.

The major work in the acute phase is to stable vital signs. Use the passive rehabilitation therapy. Passively maintain joint activities to avoid the joint contracture.

When the patient is stable, the rehabilitation treatment is the next step. Patients have about three months of spontaneous recovery of nerve. Some lost function of the body will be recovered in this stage. Therefore, at this point rehabilitation will be very important to prime time

According to doctors' suggestion, the patients of infarction stroke can start their rehabilitation after 3 5 days after onset. The hemorrhage patients should start their rehabilitation after 7 days after onset. The rehabilitation treatment can be applied in the early stage after onset. In the acute phase, the treatment is passive prevention. When the patient is stable, the treatments are actively induced and functional training. However, the rehabilitation ward beds are difficult to get. Hence, the patients often miss the recovery of prime time.

To overcome this inconvenience, a simple wearable rehabilitation robotics is developed in this manuscript. The patients can finish the motion of rehabilitation by wearing the robotics to drive the elbow and limbs. To avoid the sports injuries, the EMG is used to achieve the intelligent function. By analyzing the signal of EMG, the robotics can automatically tune the angle between the motions of stretching and bending. 


\section{LITERATURE REVIEWING}

Rehabilitation is an important topic in medical area. There are many articles talking about the robotics help rehabilitation process. Dr. Lum compared the treatment by robotics with the traditional neurodevelopmental treatment [1]. The effect of the robotics is significantly better than the traditional method. The robotics used in this paper just repeated the stretching and bending actions without feedback signals.

In 2003 year, Krebs and Hogan used MIT-MANUS robotics to treat patient [2-3]. The results show that it is helpful for increase the muscle power of the elbow. However, it is not helpful for finger joints.

The first generation of GENTLE robotics is developed by Goote and Stokes [4]. The research shows the robotics operated by physical therapist can reduce pain and increase mobility. Dr. O'Sulivan focuses his research on the torque effectionof applying robotics to upper and lower limbs.

\section{EMG INTRODUCTION}

The EMG signal is to measure the voltage change while the muscle systolic and diastolic. The surface EMG (SEMG) measured by two electrodes is to achieve the non-invasive measurement. The EMG signal is used to automatically tune the angle between the stretching and bending of rehabilitation robotics.

\section{A. SEMG Characteristic}

When neurons are stimulated, the contraction of muscle fibers will be produced. Different intensity of stimulation will produce different voltage. Usually, the SEMG signals like the high frequency noise. The peak-to-peak voltage is changed within $0 \sim 10 \mathrm{mV}$. The frequency varies in the ranges of $0 \sim 500 \mathrm{~Hz}$. The normal EMG looks like figure 1.

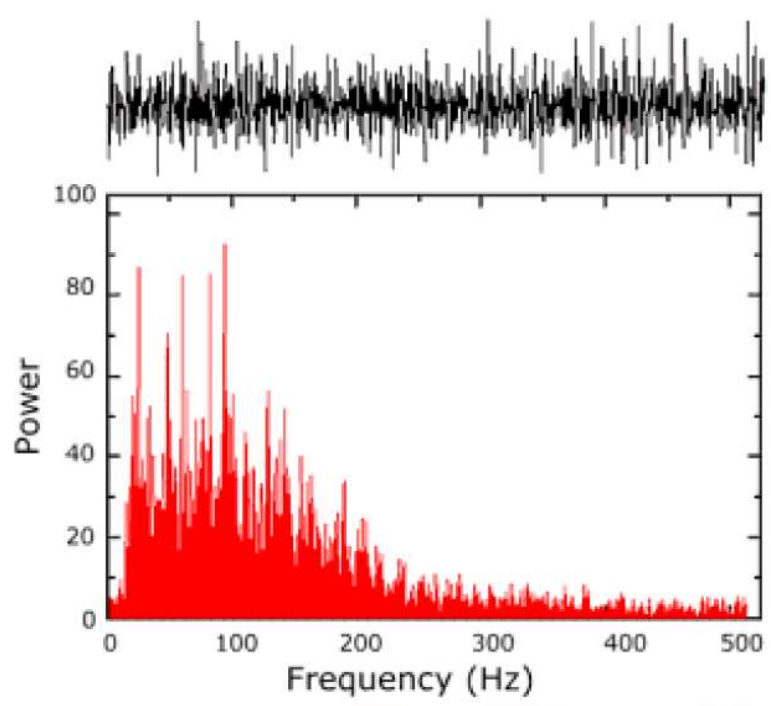

Fig 1: The maps of time domain and frequency domain of EMG [5-7]

\section{B. EMG Process}

Due to the small peak-to-peak voltage of the EMG, the characteristic of the EMG is difficult to analyze. Therefore, to apply the signals, the pre-amplifier is necessary for signal process. In this paper, a bandreject filter $(50 \sim 70 \mathrm{~Hz})$ is applied to eliminate the noise from the power line $(60 \mathrm{~Hz})$. Usually, the noise effect from the DC bias source focuses on low frequency. Hence, a band pass filter $(200 \sim 1000 \mathrm{~Hz})$ is serial connected at the next stage to extract the exact EMG signal. By the way, in order to exactly distinguish the characteristic, the filters are designed compounded with the amplifiers. The 1000 times magnification drives the peak-to-peak voltage of the $E M G$ into the range of $0 \sim 10 \mathrm{~V}$. It can efficiently eliminate the bit-error while digital process.

In this application of the EMG signals in this paper, the signal is used to decide whether the direction of stretching or bending changes or not. Hence, in this paper, the purpose is to find out the change point between the muscle stretching and bending in the time series of the EMG signals.

The EMG signals perform like a high frequency signals even after filtering. Hence, a smoothing method is also needed to smooth the high frequency signals. The rolling average method is used in this paper. Take 250 points to average from now and forward 249 points time series signals. The signals after smoothing can be shown in figure 2 . 


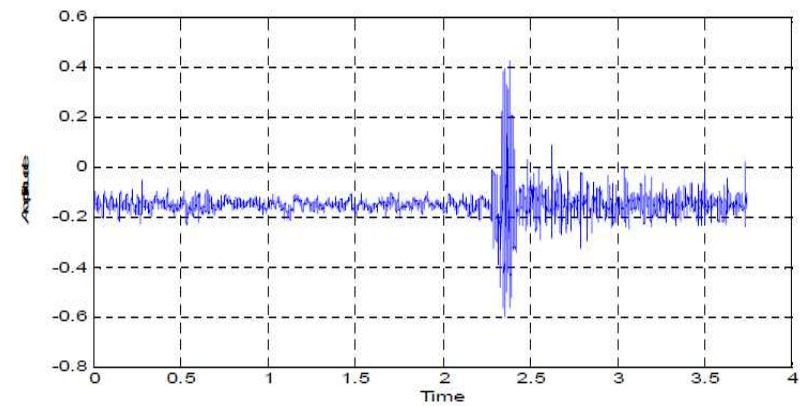

Fig 2: The EMG signals while muscle stretching and bending.

In this figure, the signals in the first time show the muscle is in relaxing situation. The peak-to-peak voltage is about $0.1 \mathrm{~V}$. At the time of 2.3 second, hand starts to clench fist to show the situation of the muscle tight. The small period of extremely oscillating signals are shown. Then, a stable oscillating signal with peak-to-peak voltage of $0.2 \mathrm{~V}$ show again. The conclusion can be obtained that the muscle after a large oscillating will stabilize within a different stable range. Therefore, by detecting the stable oscillating peak, one can tell from the muscle's situation. Then, apply the situation detecting to the rehabilitation robotics. If the muscle is in relaxing situation, the robotics can continue the stretching or bending motion. If the muscle is in tight situation, then the motion should be reverse in order to avoid the sport injuries. This will achieve the intelligent performance.

\section{REHABILITATION ROBOTICS DESIGN}

In this paper, a rehabilitation robotics is designed based on the EMG. The CompactRIO is used to perform the intelligent signal process and decision. The step motors are used to drive the robotics' joints. The detailed description is shown in the following sections.

\section{A. Mechanisms of rehabilitation robotics}

There are three parts designed in the rehabilitation robotics. They are applied to three parts of body those are shoulder, elbow and ankle. The driven unit is the step motor. Two limit switches are adapted in the stroke for safe consideration. The figure 3 shows the design draft of the shoulder part of the rehabilitation robotics. The figure 4 shows the parts of the elbow and ankle.

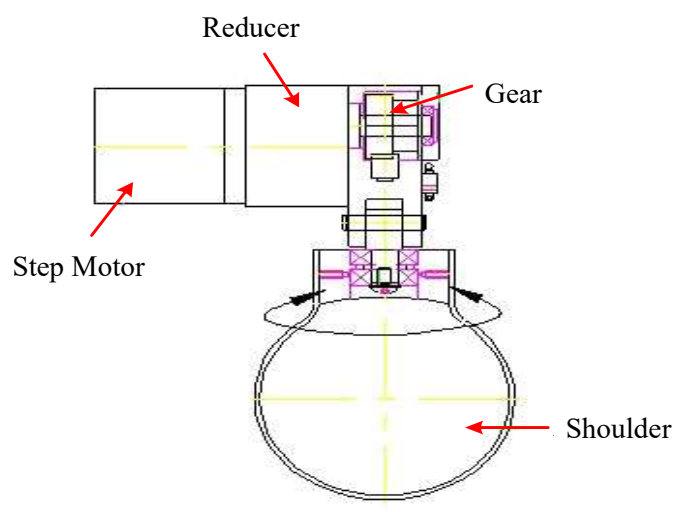

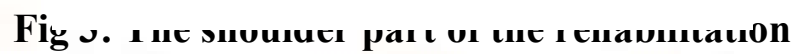
robotics
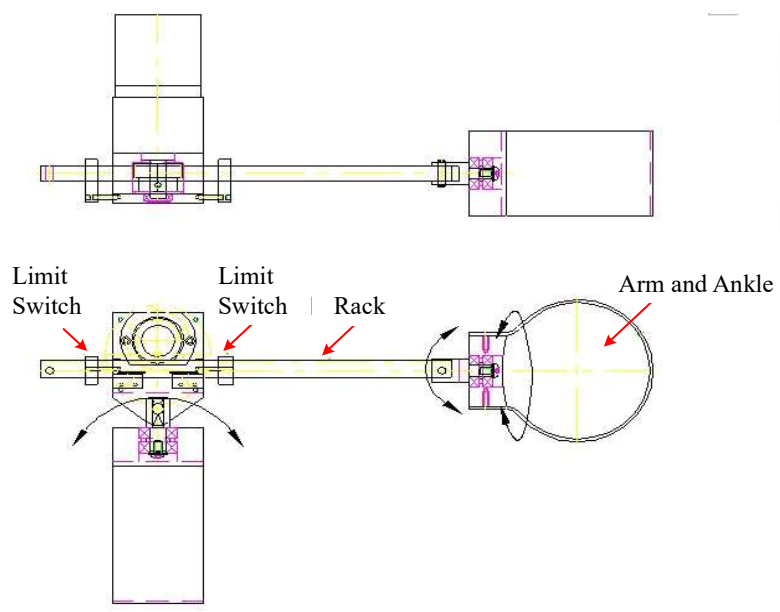

Fig 4: The parts of the elbow and ankle of the rehabilitation robotics

The physical maps of the three parts are shown in figures 5 6. The Velcro is used to fixed the robotics and the body.

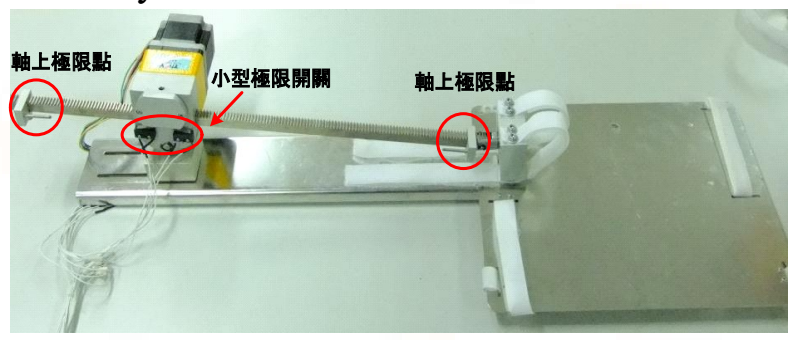

Fig. 5. The physical map of the shoulder robotics

Fig. 6. The physical map of the robotics of elbow and ankle 


\section{B. Step motor}

The driven unit in this paper is the step motor. The two phases step motor of Troy Co. is used in this paper. The model number is TK266P-02A1. The step motor design draft is shown in figure 7.

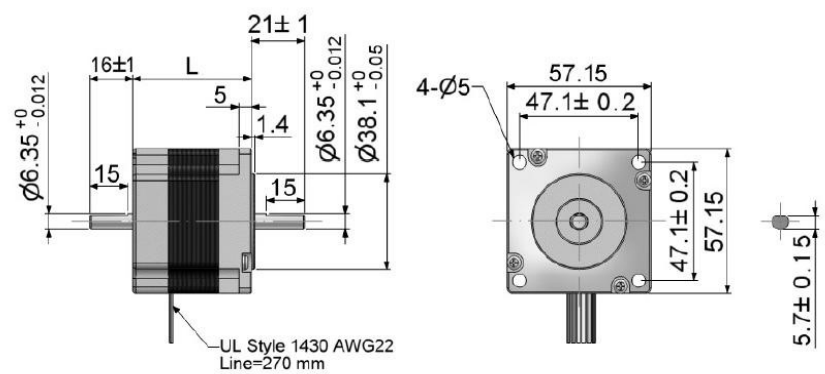

Fig. 7. The design draft of the step motor

The driver of Troy Co. with type No. TR22-1 is a high torque one axis output driver. The front sight of the driver is shown in figure 8.

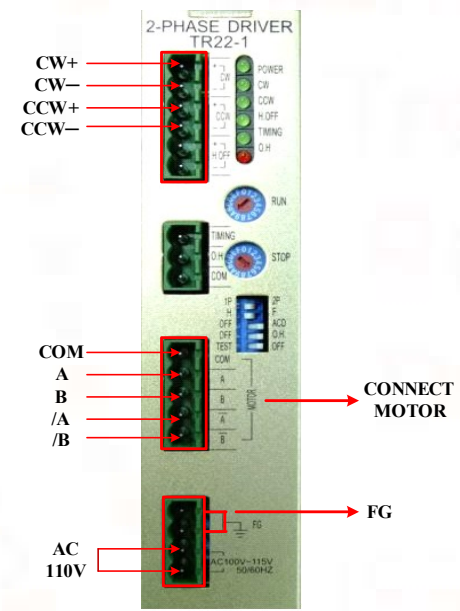

Fig. 8. The front sight of the dirver

\section{Compacr RIO}

In this paper, the CompactRIO of the NI Co. is used to process the EMG signal and make the intelligent decision. The model type cRIO 9074 is the major base unit. Based the Labview programming, a simple and powerful decision making is programmed and download into the CompactRIO. The A/D converter module of NI 9201 processes the analog EMG signal transferred to digital signal. After the intelligent decision, the digital signal for driving the step motor outputs from the digital output module NI 9403.

\section{EXPERIMENTAL RESULTS}

The CompactRIO will process the EMG signals to examine the situation of the muscle then output the signal to drive the robotics. According to the patient's physiological, the angle of the rehabilitation robotics will auto-tuning to finish the repeated stretching and bending motion.

Figure 9 shows the shoulder rehabilitation robotics wears on the patient. Figure 10 shows the robotics continuous action figures.

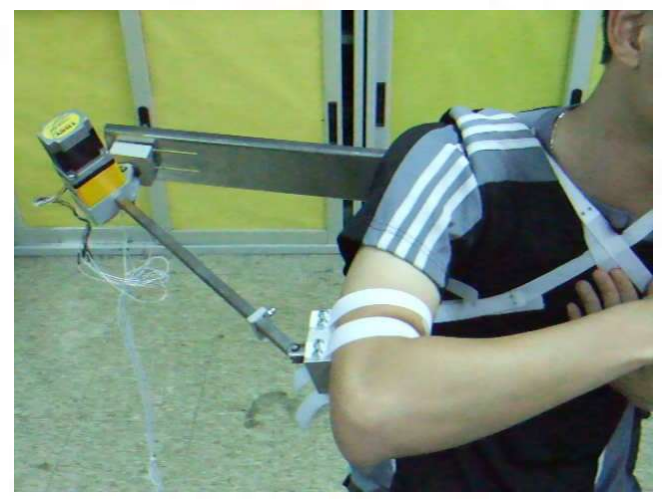

Fig 9: The patient wears the shoulder rehabilitation robotics

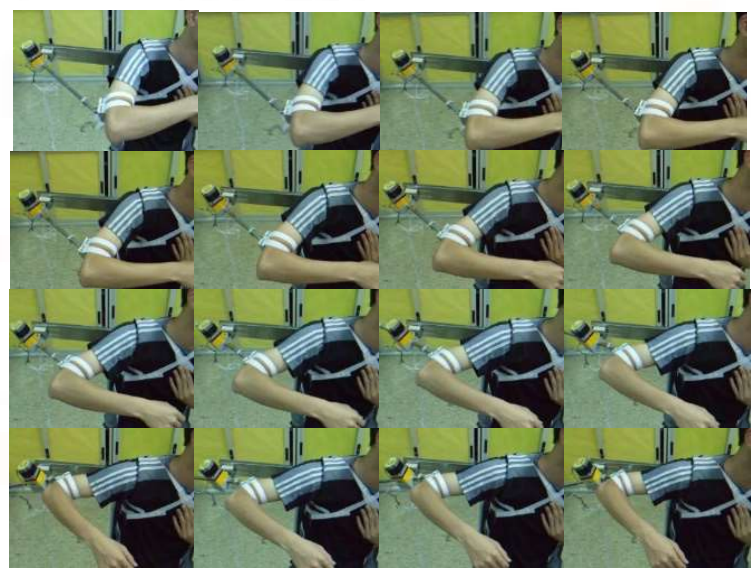

Fig 10: The continuous action figures of the shoulder rehabilitation robotics

Obviously, the rehabilitation robotics can repeat action according to the EMG signal feedback. Therefore, the men taking care of patient don't need to implement the rehabilitation movement by human force. The relative figures of the elbow part of the rehabilitation robotics are shown in figures 11 12. 


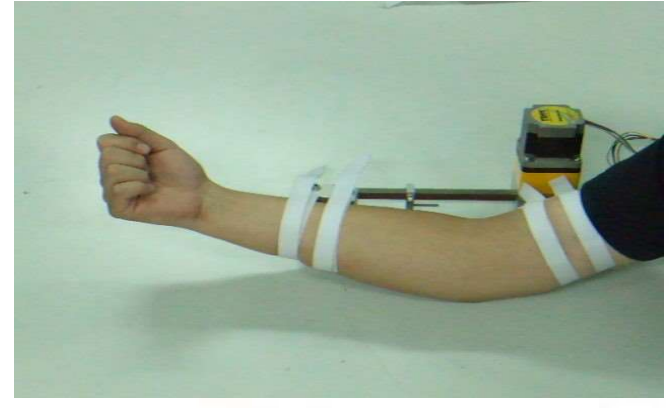

Fig 11: The patient wears the elbow rehabilitation robotics

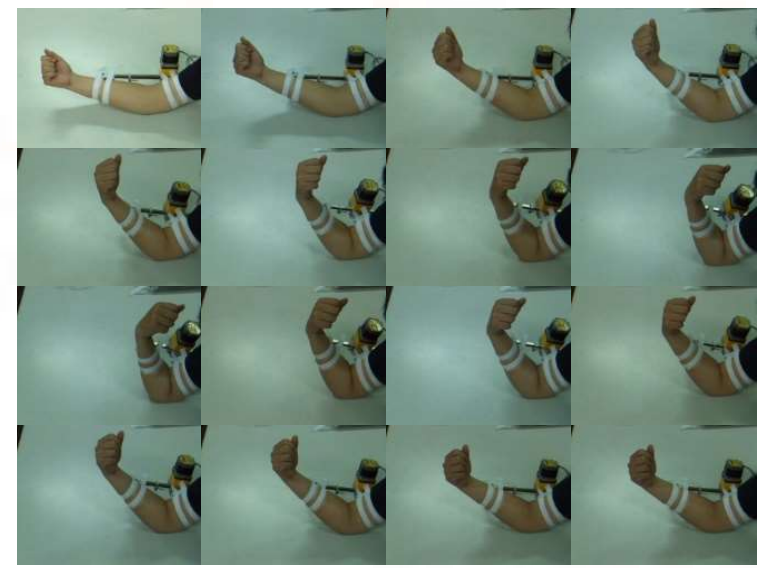

Fig 12: The continuous action figures of the elbow rehabilitation robotics

The relative figures of the ankle part of the rehabilitation robotics are shown in figures 13 14.

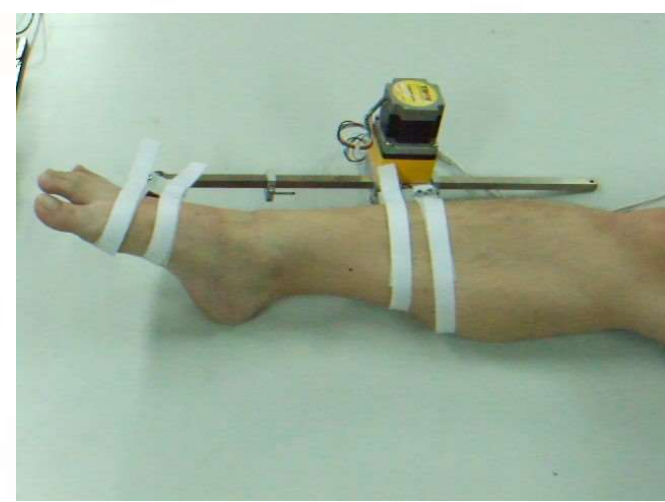

Fig 13: The patient wears the ankle rehabilitation robotics

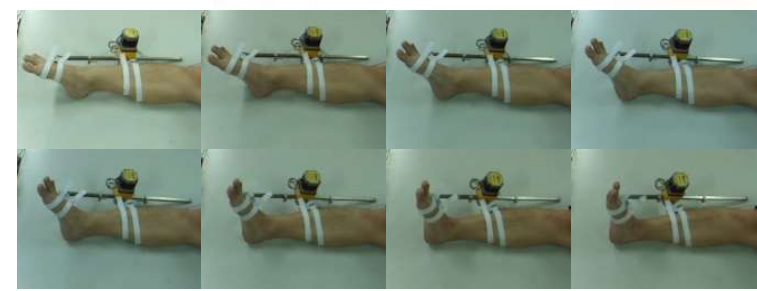

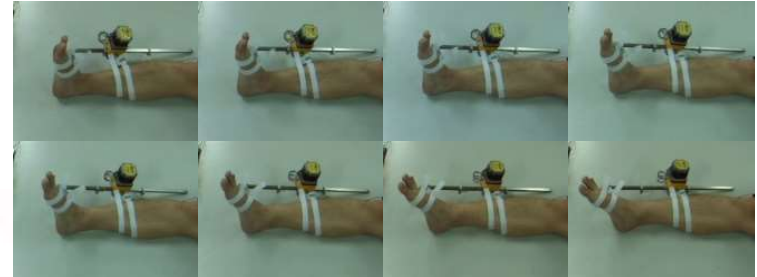

Fig 14: The continuous action figures of the ankle rehabilitation robotics

\section{CONCLUSION}

An intelligent rehabilitation robotics based on EMG is designed in this paper. Referring to the experimental results, the practice of the propose robotics is clearly. However, the weight is still a big problem up to now. Besides, the EMG signal is only used to distinguish the situation of muscle relaxing and tight. More application of the EMG signals will be more researched.

\section{REFERENCES}

[1] P. S. Lum, C. G. Burgar and P.C. Shor, "Robotassisted Movement Training Comapred with Convnetionary Therapy Technologies for the Rehabilitation of Upper-limb Function AfterStroke," Arch Phys. Med. Rehabil., pp. 952-958, 2002.

[2] H. I. Krebs, B. T. Volpe, M. L. Aisen, W. Hening, S. Admovich, H. Poizner, K. Subrahmanyan, N. Hogan, "Robotic Application in Neuromotor Rehabilitation," Robotica, pp. 3, 2003.

[3] S. E. Fasoli, H. I. Krebs, J. Stein, W. R. Frontera, N. Hogan, "Effects of Robotics Therapy on Motor Impairment and Recovery in Chronic Stroke," Arch. Phys. Med. Rehabil.,pp. 477-482, 2003.

[4] S. Coote, E. K. Stokes, "Robot Mediated Therapy: Attitudes of Patients and Therapists Towards the First Prototype of the GENTLE's System," Technology and Disability, pp. 27-34, 2003

[5] L. W. O’Sulivan, T. J. Gallwey, "Upper-limb Surface Electro-myography at Maximum Supination and Pronation Torques: the Effect of Elbow and Forearm Angle," Journal of Electromygraphy and Kinesiology, pp. 275-285, 2002.

[6] U. Noriyoshi, "EMG Pattern Recognition by Neural Networks for Multi Fingers Control," Engineering in Medicine and Biology Society, Proceedings of the Annual International Conference, pp. 1-2, 1992.

[7] http://www.delsys.com/emg_articles/EMG.shtm 